Artikel

\title{
Analysis and Implementation of Online-Based Rice Sales (e-commerce) at UD. Toko Beras Santy
}

\author{
Dram Renaldi ${ }^{1}$, Tomi Gunawan ${ }^{2}$, \\ ${ }^{1,2}$ Buddhi Dharma University, Information System, Banten, Indonesia
}

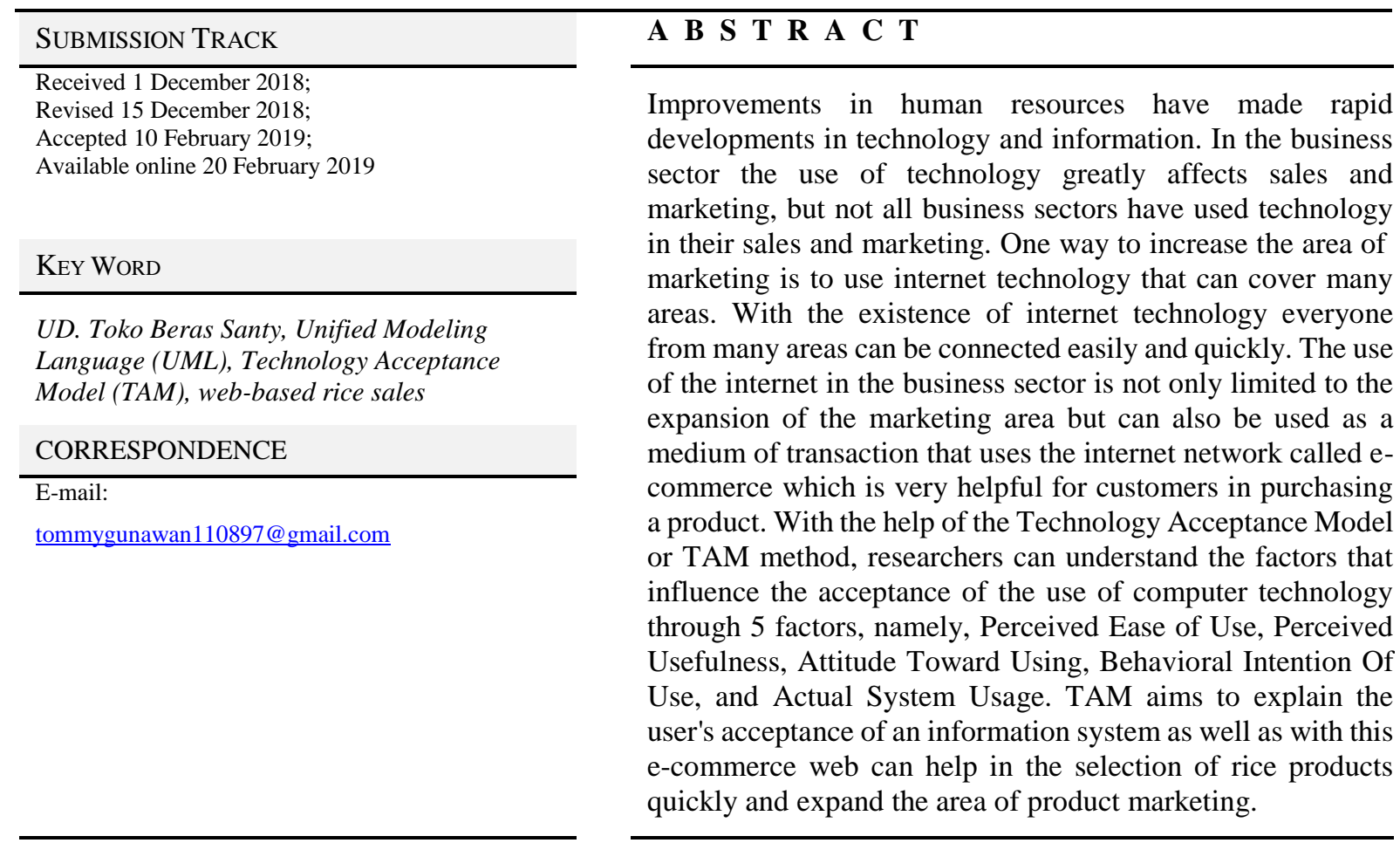

\section{INTRODUCTION}

The development of Information Technology which is accompanied by the development of the internet support each other so that it makes a concept of Information Technology, in the business sector, the development of technology plays an important role and gives a very significant impact on the systems that work in that business field, especially in sales, processing data and service improvements provided to customers. With the existence of internet technology all people from many regions can be connected easily and quickly, the use of internet technology is not only limited to the utilization and dissemination of information between people, but can also be used as a medium or a means to conduct a trade transaction called e-commerce. Ecommerce or Electronic Commerce is a form of electronic transactions of sales, purchases and payments using internet network media, according to Munawar (2009), E-commerce is a business process using electronic technology that connects companies, consumers and the public in the form of Electronic transactions, sales of goods, and information electronically. While according to Jony Wong (2010), E-commerce is a business transaction that occurs in electronic networks such as the internet. At UD. Toko Beras Santy, the absence of online marketing makes it difficult for buyers to see directly the 
rice products that are available in addition to having to come to the store, and because they have not used online marketing done by UD. Santy's Rice Shop does not yet cover a large area. Based on the background contained above, a study was conducted under the title "Analysis and Implementation of OnlineBased Sales (E-commerce) at UD.Toko Beras Santy "using the PHP programming language and using mySql as a database.

\section{METHOD}

The method used in this study includes two parts, method of collecting data and testing methods.

\section{Method of Collecting Data}

\section{a. Observation}

Observation, which is to make direct observations on the relevant business premises and collect information needed to help design the system

b. Interview

After getting the data and information needed in the previous stage, then do a question and answer directly with the parties involved at UD.Toko Beras Santy, with the aim of obtaining data directly from related sources.

\section{c. Literature review}

Search for information needed to design web-based information systems by reading and studying them from existing books and other sources. In writing this thesis the author collected data that is very useful for the information provided by this application sourced from books in the company and library as a reference

\section{d. Questionnaire}

Collecting data to customers by giving a questionnaire in the form of questions about rice products. Data collection method by giving questions to 30 customers, to determine how useful applications have been made using the grid on the TAM testing method.

\section{Testing Method}

The testing method used in this e-commerce application is using the Technology acceptance model (TAM) method:

Technology Acceptance Model (TAM) is a model created to analyze and understand the factors that influence the acceptance of a computer technology in an organization or end user. The Technology Acceptance Model (TAM) was first introduced by a scientist named Davis M (1986). The purpose of the Technology Acceptance Model (TAM) itself is to explain and predict user acceptance of an information system technology. There are five attitudes forming that affect a person's behavior in the use of information system technology, namely:

\section{a. Perceived Ease Of Use}

Convincing the user or end user that information technology is easy to use and operate

b. Perceived Usefullnes

Convincing the user or end user that the information technology used will provide many beneficial and promising benefits.

c. Attitude Toward Using

Convincing the user's attitude to use and operate the information technology that is used.

\section{d. Behavioral Intention Of Use}

Improve and maximize user behavior to always continue to use the information technology provided.

\section{e. Actual system Usage}

Stating that the user has used the information technology to the fullest and is based on the benefits to be gained. 
Here is a diagram of the Technology Acceptance Model

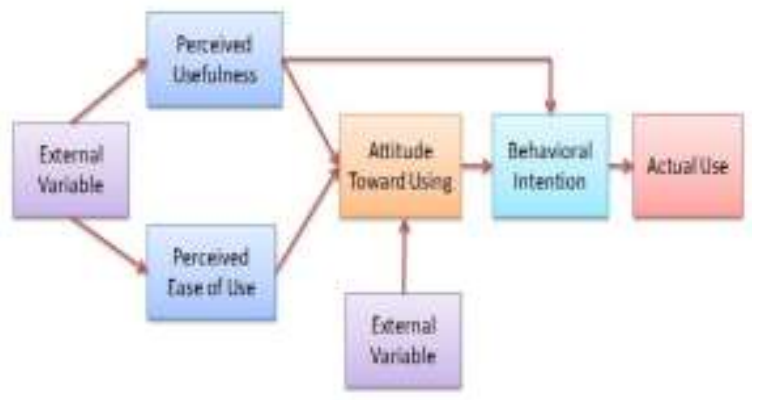

Fig 1. TAM Diagram

\section{RESULTS}

After conducting research on UD. Toko Beras Santy, then made a solution in the form of ecommerce selling rice based online using the Technology Acceptance Model testing method, the initial step in this research is to create a questionnaire instrumentation that is made using open question, respondents will fill in the questionnaire answers and directly analyzed using IBM SPSS Statistics. Here are the grids for questions:
Table 1. Questionnaire grid

\begin{tabular}{|c|c|c|c|}
\hline No & Variabel & Indikator & Jumlah \\
\hline \multirow[t]{4}{*}{1} & \multirow[t]{4}{*}{ CSE } & $\begin{array}{l}\text { Dapat berhasil memecahkan } \\
\text { masalah berkomputer }\end{array}$ & \\
\hline & & $\begin{array}{l}\text { Tahu bagaimana menangani } \\
\text { situasi yang tak terduga } \\
\text { berkomputer }\end{array}$ & \\
\hline & & $\begin{array}{l}\text { Selalu bersikap tenang dalam } \\
\text { menghadapi } \\
\text { berkomputer }\end{array}$ & \\
\hline & & $\begin{array}{l}\text { Dapat menemukan solusi dalam } \\
\text { permasalahan berkomputer }\end{array}$ & \\
\hline \multirow[t]{2}{*}{2} & \multirow[t]{2}{*}{ PEOU } & Aplikasi Mudah di gunakan & \\
\hline & & $\begin{array}{l}\begin{array}{l}\text { Aplikasi mudah untuk di } \\
\text { navigasikan }\end{array} \\
\end{array}$ & \\
\hline \multirow[t]{4}{*}{3} & \multirow[t]{4}{*}{ PU } & Membantu lebih cepat & \\
\hline & & Membantu lebih akurat & \\
\hline & & Menjadi lebih efektif & \\
\hline & & Menjadi lebih mudah & \\
\hline \multirow[t]{4}{*}{4} & \multirow[t]{4}{*}{ ATU } & Senang & \\
\hline & & Percaya & \\
\hline & & Puas & \\
\hline & & Memilih untuk menggunakan & \\
\hline \multirow[t]{4}{*}{5} & \multirow[t]{4}{*}{ ITU } & Bertujuan untuk digunakan selalu & \\
\hline & & $\begin{array}{l}\text { Menggunakan dalam } 20 \text { menit } \\
\text { sekali pakai }\end{array}$ & \\
\hline & & $\begin{array}{l}\text { Berkomunikasi melalui media } \\
\text { website }\end{array}$ & \\
\hline & & $\begin{array}{lll}\begin{array}{l}\text { Bergabung } \\
\text { pengguna }\end{array} & \text { dalam } & \text { forum } \\
\end{array}$ & \\
\hline \multirow[t]{4}{*}{6} & \multirow[t]{4}{*}{$\mathrm{AU}$} & Memahi cara dalam penggunaan & \\
\hline & & $\begin{array}{l}\text { Bersedia bertanya } \\
\text { melakukan diskusi }\end{array}$ & \\
\hline & & $\begin{array}{l}\text { Bersedia menyampaikan } \\
\text { kepuasaan penggunaan }\end{array}$ & \\
\hline & & $\begin{array}{l}\begin{array}{l}\text { Membantu rekan dan teman } \\
\text { dalam } \\
\text { websitetersebut. }\end{array} \\
\text { menggunakan }\end{array}$ & \\
\hline
\end{tabular}

After obtaining the answers to the questionnaire from the customer and admin UD. Toko Beras Santy, an analysis using IBM SPSS Statistics to find out whether the technology acceptance answers are valid or not, here are some test results: 
a. Data Correlation Test Results

Table 2.Data Correlation Test

Correlations

\begin{tabular}{|c|c|c|c|c|c|c|c|c|c|}
\hline & & PEOU 1 & PEOU 2 & PU 1 & $\mathrm{PU} 2$ & PU 3 & ITU 1 & ITU 2 & ITU 3 \\
\hline \multirow[t]{3}{*}{ PEOU 1} & $\begin{array}{l}\text { Pearson } \\
\text { Correlation }\end{array}$ & 1 & $.793^{* *}$ & $.489^{* *}$ & .338 & $.311^{*}$ & $.558^{* *}$ & $.558^{\star *}$ & $.636^{* *}$ \\
\hline & Sig. (1-tailed) & & .000 & .003 & .233 & .047 & .001 & .001 & .000 \\
\hline & $\mathrm{N}$ & 30 & 30 & 30 & 30 & 30 & 30 & 30 & 30 \\
\hline \multirow[t]{4}{*}{ PEOU 2} & Pearson & $793^{* *}$ & 1 & $272^{*}$ & 375 & $152^{* *}$ & $592 *$ & $12 \mathrm{Q}^{\star *}$ & $180^{* \star}$ \\
\hline & Correlation & .195 & 1 & .373 & .315 & $.45<$ & .596 & $.4 \angle 0$ & .409 \\
\hline & Sig. (1-tailed) & .000 & & .021 & .346 & .006 & .000 & .009 & .003 \\
\hline & $\mathrm{N}$ & 30 & 30 & 30 & 30 & 30 & 30 & 30 & 30 \\
\hline \multirow[t]{4}{*}{ PU 1} & Pearson & $180^{* *}$ & $272^{*}$ & 1 & $52 \Omega^{\star *}$ & 177 & $200 *$ & م & $500 * \star$ \\
\hline & Correlation & .409 & .013 & 1 & .530 & .171 & .309 & .309 & .000 \\
\hline & Sig. (1-tailed) & .003 & .021 & & .001 & .175 & .049 & .049 & .001 \\
\hline & $\mathrm{N}$ & 30 & 30 & 30 & 30 & 30 & 30 & 30 & 30 \\
\hline \multirow[t]{4}{*}{ PU 2} & Pearson & & & $500^{* *}$ & & & & & \\
\hline & Correlation & .000 & (נ) & .030 & 1 & .001 & .310 & .318 & $.45<$ \\
\hline & Sig. (1-tailed) & .233 & .346 & .001 & & .189 & .123 & .123 & .006 \\
\hline & $\mathrm{N}$ & 30 & 30 & 30 & 30 & 30 & 30 & 30 & 30 \\
\hline \multirow[t]{4}{*}{ PU 3} & Pearson & $211^{*}$ & $152^{* *}$ & דרפ & 267 & 1 & $502^{* *}$ & $500^{* *}$ & $150^{* *}$ \\
\hline & Correlation & (311 & .452 & $.27 \pi$ & .267 & 1 & .582 & .582 & .452 \\
\hline & Sig. (1-tailed) & .047 & .006 & .175 & .189 & & .000 & .000 & .006 \\
\hline & $\mathrm{N}$ & 30 & 30 & 30 & 30 & 30 & 30 & 30 & 30 \\
\hline \multirow[t]{4}{*}{ ITU 1} & Pearson & $558^{* *}$ & $592^{* *}$ & $309^{*}$ & 318 & $582^{\star *}$ & 1 & $603^{* *}$ & $502^{* *}$ \\
\hline & Correlation & .530 & .092 & .309 & .318 & .582 & 1 & .683 & .592 \\
\hline & Sig. (1-tailed) & .001 & .000 & .049 & .123 & .000 & & .000 & .000 \\
\hline & $\mathrm{N}$ & 30 & 30 & 30 & 30 & 30 & 30 & 30 & 30 \\
\hline \multirow[t]{4}{*}{ ITU 2} & Pearson & $558^{\star *}$ & $428^{* *}$ & $309^{*}$ & 218 & $582^{* *}$ & $683^{* *}$ & 1 & $757^{* *}$ \\
\hline & Correlation & & & & & & .000 & 1 & \\
\hline & Sig. (1-tailed) & .001 & .009 & .049 & .123 & .000 & .000 & & .000 \\
\hline & $\mathrm{N}$ & 30 & 30 & 30 & 30 & 30 & 30 & 30 & 30 \\
\hline \multirow[t]{4}{*}{ ITU 3} & Pearson & $62 a^{* *}$ & 180 ** & {$\left[22^{* *}\right.$} & $152^{* *}$ & $150^{\star *}$ & $502^{* *}$ & & 1 \\
\hline & Correlation & .036 & .489 & .533 & .452 & .452 & .592 & .157 & 1 \\
\hline & Sig. (1-tailed) & .000 & .003 & .001 & .006 & .006 & .000 & .000 & \\
\hline & $\mathrm{N}$ & 30 & 30 & 30 & 30 & 30 & 30 & 30 & 30 \\
\hline
\end{tabular}

Testing the Quality of Data on questionnaire answers using IBM SPSS Statistics. With a standard coefficient above 0.3 . Where there is a correlation coefficient of 0.3 or more, the data is declared valid. 
b. Reliability Test Results

Table 3. Reliability Test Results

\begin{tabular}{|c|c|c|c|c|}
\hline & $\begin{array}{l}\text { Scale Mean if } \\
\text { Item Deleted }\end{array}$ & $\begin{array}{l}\text { Scale Variance } \\
\text { if Item Deleted }\end{array}$ & $\begin{array}{l}\text { Corrected Item- } \\
\text { Total Correlation }\end{array}$ & $\begin{array}{c}\text { Cronbach's } \\
\text { Alpha if Item } \\
\text { Deleted }\end{array}$ \\
\hline PEOU 1 & 22.87 & 5.016 & .706 & .841 \\
\hline PEOU 2 & 22.97 & 5.275 & .643 & .849 \\
\hline PU 1 & 22.90 & 5.403 & .526 & .862 \\
\hline PU 2 & 23.03 & 5.964 & .340 & .878 \\
\hline PU 3 & 23.03 & 5.620 & .529 & .861 \\
\hline ITU 1 & 22.93 & 5.099 & .708 & .841 \\
\hline ITU 2 & 22.93 & 5.099 & .708 & .841 \\
\hline ITU 3 & 22.97 & 4.999 & .798 & .831 \\
\hline
\end{tabular}

\section{Reliability Statistics}

\begin{tabular}{|r|r|}
\hline $\begin{array}{c}\text { Cronbach's } \\
\text { Alpha }\end{array}$ & N of Items \\
\hline .867 & 8 \\
\hline
\end{tabular}

Based on the results of the Reliability Test table contained above, it can be said that this data is all reliable because the Cronbach Alpha value is above 0.4.

c. F Test Results

Table 4. F Test Results

\begin{tabular}{|ll|r|r|r|r|r|}
\hline \multicolumn{1}{|c|}{ ANOVA $^{\mathrm{a}}$} & \\
\hline 1 & Sum of Squares & \multicolumn{1}{c|}{$\mathrm{df}$} & Mean Square & \multicolumn{1}{c|}{$\mathrm{F}$} & \multicolumn{1}{c|}{ Sig. } \\
\hline & Regression & 2.344 & 2 & 1.172 & 7.998 & $.002^{\mathrm{b}}$ \\
& Residual & 3.956 & 27 & .147 & & \\
& Total & 6.300 & 29 & & & \\
\hline
\end{tabular}
a. Dependent Variable: ITU 1
b. Predictors: (Constant), PEOU 2, PEOU 1

The table above shows that the F value is 7.998 with a significance level of 0.002 . With this data it can be shown that the PEOU and PU variables affect the use of the Toko Beras Santy's 
web, in the sense that users are easy to use the Toko Beras Santy's web and it is expected that users will be comfortable using it.

d. T Test Results

Table 5. T Test Results

\begin{tabular}{|c|c|c|c|c|c|c|}
\hline \multicolumn{7}{|c|}{ Coefficients $^{a}$} \\
\hline \multirow{2}{*}{\multicolumn{2}{|c|}{ Model }} & \multicolumn{2}{|c|}{ Unstandardized Coefficients } & \multirow{2}{*}{$\begin{array}{c}\text { Standardized } \\
\text { Coefficients } \\
\text { Beta }\end{array}$} & \multirow[b]{2}{*}{$\mathrm{t}$} & \multirow[b]{2}{*}{ Sig. } \\
\hline & & $\mathrm{B}$ & Std. Error & & & \\
\hline \multirow[t]{3}{*}{1} & (Constant) & 1.171 & .537 & & 2.181 & .038 \\
\hline & PEOU 1 & .228 & .238 & .240 & .959 & .346 \\
\hline & PEOU 2 & .417 & .259 & .402 & 1.608 & .120 \\
\hline
\end{tabular}

a. Dependent Variable: ITU 1

The PEOU variable has a $\mathrm{T}$ value of 2.181 with a significance level of 0.038 , because a significance value below 0.5 can be interpreted that the PEOU variable influences the web reception of Santy Rice Shop. Users have a perception that the Santy Rice Shop web is easy to operate and the user will be easier to accept the Santy Rice Shop web. It can be said that the PEOU variable greatly affects the Santy Rice Shop website by the user.

\section{DISCUSSION}

After searching for information through interviews and requirements

elicitation, the authors pour the data that has been obtained into an online rice sales application (e-commerce) that makes it easy to market rice products and makes it easy for customers to buy rice easily and quickly.
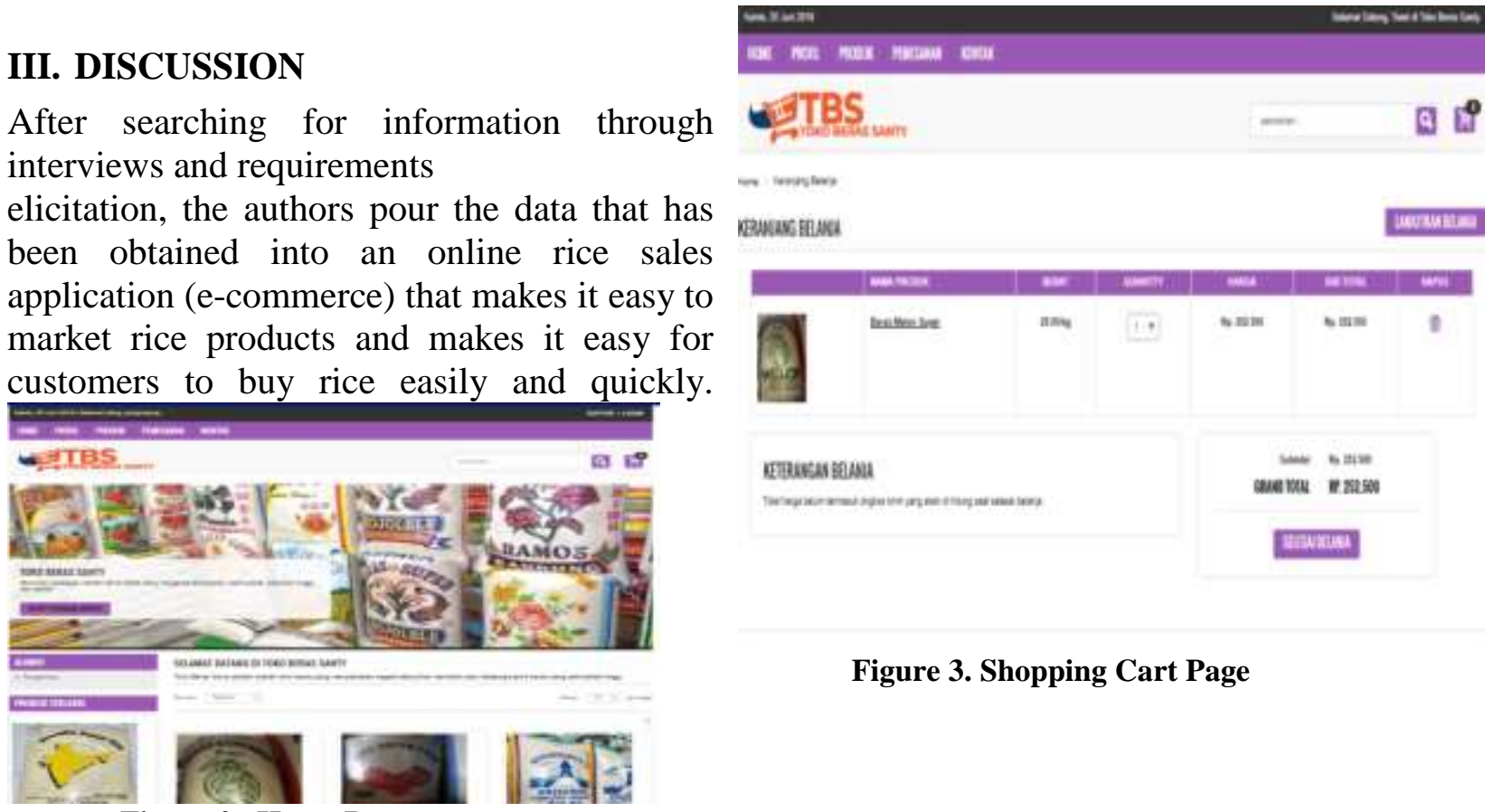

Figure 3. Shopping Cart Page

Figure 2 . Home Page 


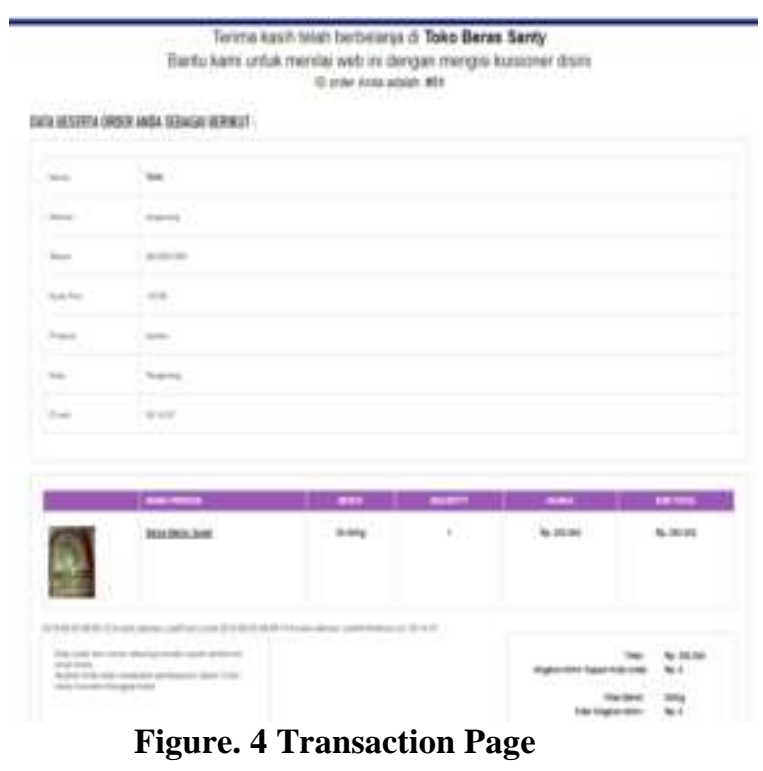

On the page above shows the page after making a transaction which also links to the TAM questionnaire to assist in testing the system.

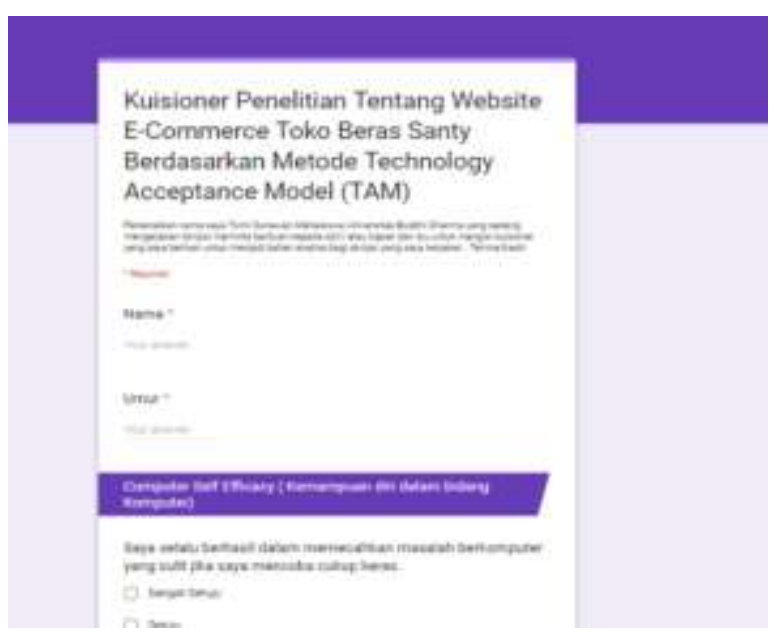

Figure 5. TAM Questionnaire Form

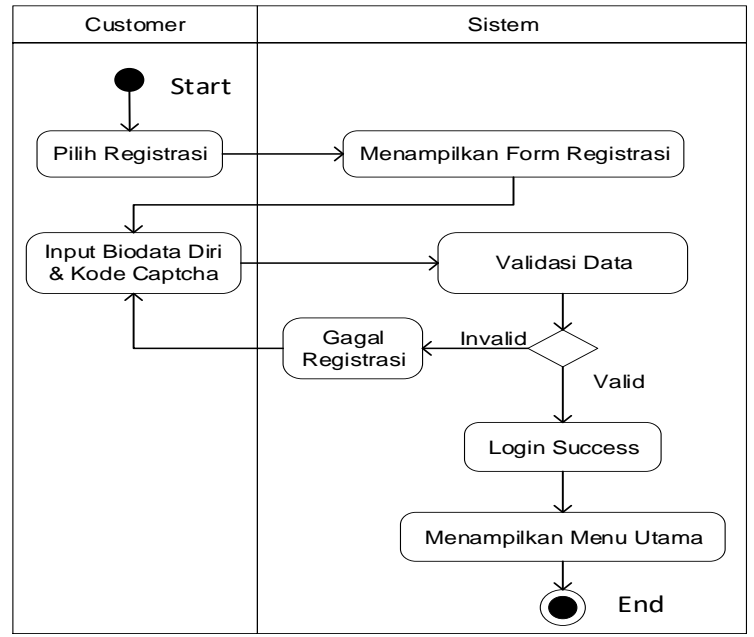

Figure 6. Activity Diagram Register

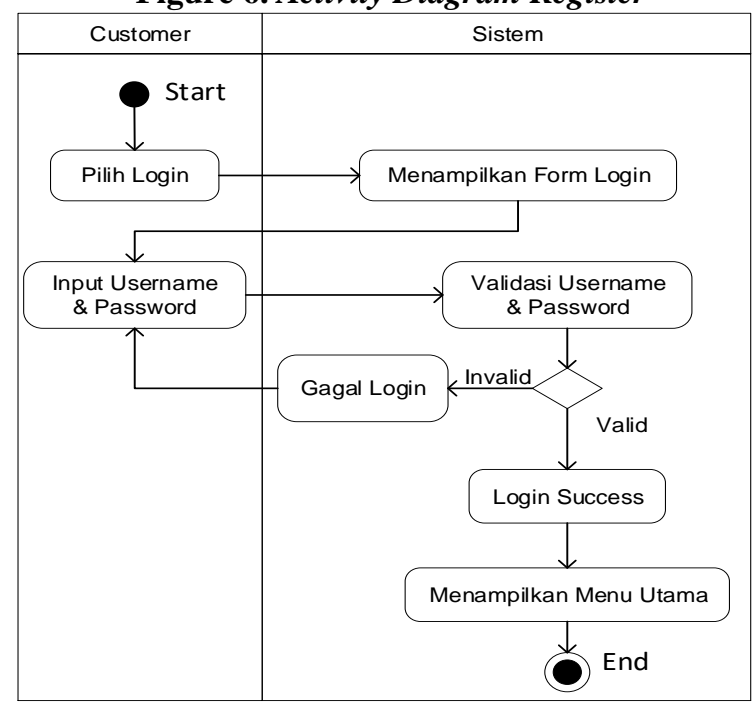

Figure 7.Activity Diagram Login 


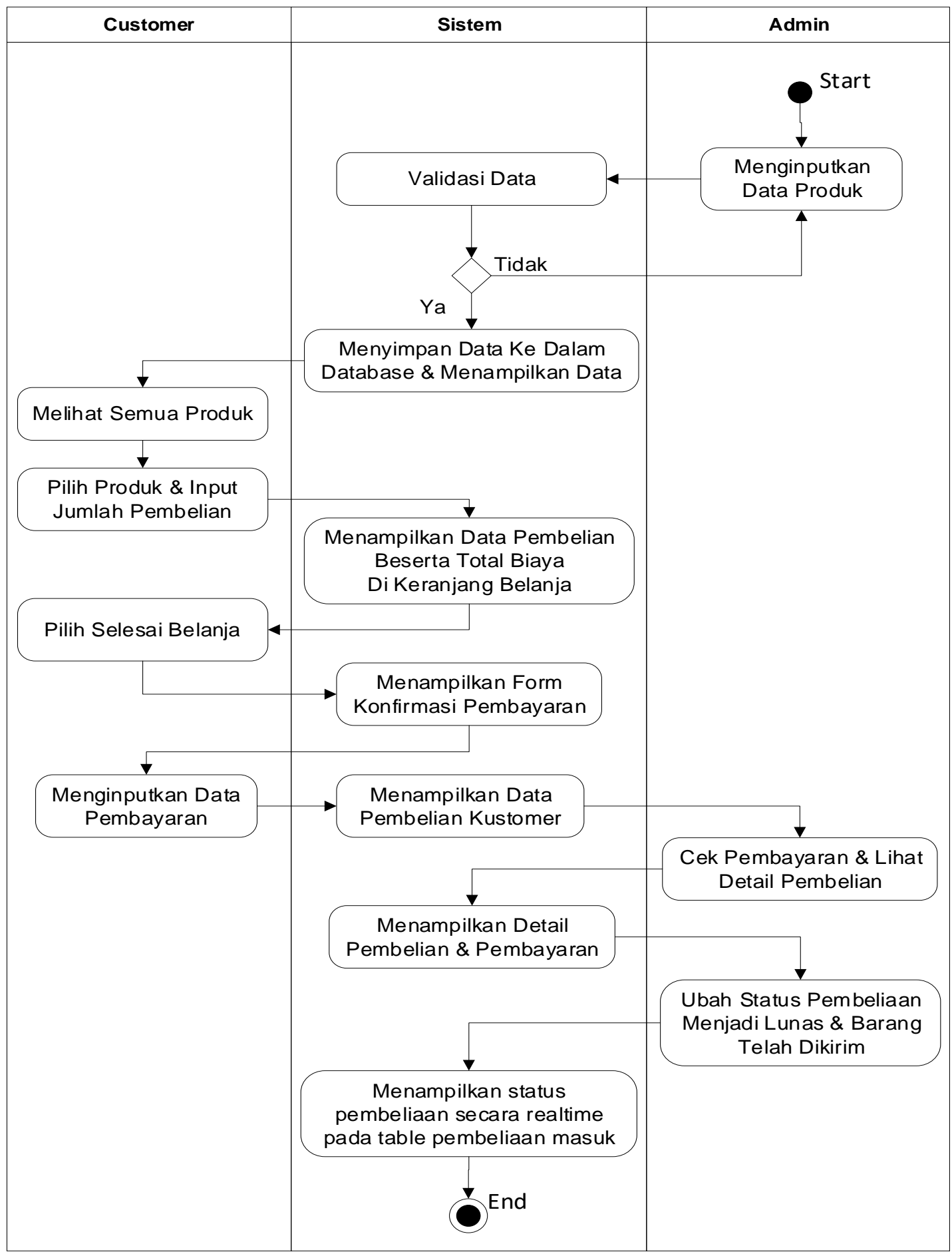

Figure 8. Activity Diagram Pembelian

The picture above is a picture of the activity diagram found in the online rice sales (e-commerce) application for UD. Toko Beras Santy. 


\section{CONCLUSION}

From the results of discussions on web-based online sales systems (e-commerce) can be drawn conclusions, namely:

1. With this e-commerce web, it can be used directly by customers to help speed up the selection of rice products according to customer criteria and desires.

2.This online sales web is also very helpful in marketing rice products in a wide range of areas.

3.By using the Technology Acceptance Model (TAM) method, can find out the results in the acceptance of e-commerce technology to customers UD. Toko Beras Santy. 


\section{REFERENCES}

Anggadini, Sri Dewi. (2011). Sistem Informasi Akuntansi. Yogyakarta, Graha Ilmu.

Davis,Gordon B .(2013). Kerangka Dasar Sistem Informasi Manajemen, Palembang, Maxikom.

Harjono . (2009) Mendayagunakan Internet, Online

Jogiyanto. (2010), Analisis dan desain Sistem Informasi, Yogyakarta , Andi Offset.

Kadir,Abdul . (2009), Membuat Aplikasi web dengan PHP + Database MySQL, Yogyakarta, Andi.

Laudon, (2012). Management System:Managing the Digital Firm Twelfth Edition. New Jersey, Prentice Hall.S

Mulyanto,Agus. (2009), Sistem Informasi Konsep dan Aplikasi, Yogyakarta, Pustaka Pelajar.

Mustakini, Jogiyanto Hartono . (2009). Sistem Informasi Teknologi, Yogyakarta, Andi Offset.

Nugroho, Adi. (2010), Rekayasa Perangkat Lunak Berbasis Objek dengan Metode USDP, Yogyakarta , Andi

Purwanto,Erwan. (2012), Implementasi Kebijakan Publik. Konsep dan Aplikasinya di Indonesia, Yogyakarta, Gava Media.

Sibero, Alexander F.K . (2011), Kitab Suci Web Programming, Yogyakarta, Mediakom. Sinar Baru.

Soemarso . (2009), Akuntansi Suatu Pengantar, Buku ke 2 Edisi 5, Jakarta, Salemba Empat.

Solichin, Ahmad. (2016), Pemrograman Web Dengan Php Dan Mysql, Hal 10, Budi Luhur.

Sujarweni, V. Wiratna. (2015). Akuntansi Manajemen. Yogyakarta, Pustaka Baru Press

Sulistiyowati, Leny. (2010).Panduan Praktis Memahami Analisis Laporan Keuangan, Jakarta, PT.Elex Media Komputindo

Sutabri, Tata. (2012), Analisis Sistem Informasi, Yogyakarta, Andi Offset

Sutarman , M.Kom.. (2012).Analisis Sistem Informasi, Yogyakarta, Andi Offset.

Sutarman. (2012), Pengantar Teknologi Informasi Jakarta, PT. Bumi Aksara. 
Widodo , Prabowo.P.Dkk . (2011), Pemodelan Sistem Berorientasi Obyek dengan UML , Yogyakarta, Graha Ilmu.

Wong,Jony . (2010), Internet Marketing for Beginners, Jakarta, Elex Media Komputindo.

Yakub.(2012). Pengantar Sistem Informasi, Yogyakarta, Graha Ilmu.

Handayani,Sutri.(2018),'Perancangan Sisitem Informasi Penjualan Berbasis E-Commerce Studi Kasus Toko Kun Jakarta', ILKOM Jurnal Ilmiah, Volume 10 Nomor 2

Irawan,Dedi.Yoyoeng Rahsel.Taufik Udin,(2017),' Perancangan Electronic Commerce Berbasis B2C Pada Toko ATK Sindoro', Jurnal TAM (Technology Acceptance Model), Volume 8 Nomor 1

Uska, Muhammad Zamroni. (2017),' Analisis Penerimaan Digital Library Menggunakan Technology Acceptance Model (TAM) Di Universitas Hamzan Wadi', Jurnal Pendidikan Informatika (EDUMATIC), Volume 1, Nomor 1

\section{BIOGRAPHY}

Dram Renaldi, was born in the city of Tangerang on January 11, 1990. Completed his undergraduate education (S.Kom.) at STMIK PGRI Tangerang in 2012 and his postgraduate education (M.Kom.) at Budi Luhur University in 2016. Now active as a permanent lecturer and chairman Software Engineering study program at the Dharma Buddhi University.

Tomi Gunawan, born in the city of Tangerang on August 11, 1997. Completed a high school education at the SMA Perguruan Buddhi in 2015. 